Research Paper

\title{
Persistence of CD34 Stem Marker in Human Lipoma: Searching for Cancer Stem Cells
}

\author{
Barbara Zavan ${ }^{2 *}$, Francesco De Francesco ${ }^{1 * \Xi}$, Francesco D' Andrea ${ }^{1}$, Letizia Ferroni ${ }^{2}$, Chiara Gardin $^{2}$, Rosa \\ Salzillo' ${ }^{1}$, Gianfranco Nicoletti ${ }^{1}$, Giuseppe A. Ferraro ${ }^{1}$ \\ 1. Multidisciplinary Department of Medical-Surgical and Dental Specialties, Second University of Naples, L. De Crecchio, 6, 80138, Naples (Italy) \\ 2. Department of Biomedical Sciences, University of Padua, Ugo Bassi, 58/B, 35131, Padua (Italy) \\ * These authors contributed equally to the work
}

$\square$ Corresponding author: Francesco De Francesco, Second University of Naples, School of Medicine and Surgery, Multidisciplinary Department of Medical-Surgical and Dental Specialties, Second University of Naples, L. De Crecchio 6, 80138 Naples, Italy; Tel.: 0039815665473; Fax.: 0039815665011; e-mail: fran.defr@libero.it; gaferraro@libero.it

(c) 2015 Ivyspring International Publisher. Reproduction is permitted for personal, noncommercial use, provided that the article is in whole, unmodified, and properly cited. See http://ivyspring.com/terms for terms and conditions.

Received: 2015.02.20; Accepted: 2015.05.05; Published: 2015.07.18

\begin{abstract}
Background: Lipomas are benign solid tumours that develop in soft tissues with origin in mesenchymal progenitors. Macroscopically, they appear as soft-elastic nodules, varying in volume from a few millimiters to several centimetres and can enlarge progressively. Although they are usually asymptomatic, they can cause symptoms due to nerve or vessel compression. Microscopically they appear as fibrous connective tissue stroma with embedded adipocytes, and absence of inflammation. Up to now no characterisation of stem cell population present in this tissue has been performed.

Methods: Cytofluorimetric, biological and molecular biology analyses have been performed in order to test superficial cell markers and gene expression profile related to stemness and apoptotic activity of cells present in lipoma tissues compared to those of adipose tissue's cells.

Results: Our results confirmed that $\mathrm{CD} 34^{+}$cells in lipoma were present around small adipocytes, showing several altered biological activity such as proliferation, apoptotis and stemness.

Conclusions: The data emerging from the comparison of the lipoma cells and normal adipose tissue, suggests the presence of cell precursors involved in the development of the lipoma. This hypothesis requires further investigation and may indicate new thresholds in the study of benign tumour pathogenesis.
\end{abstract}

Key words: CD34, lipoma, cancer stem cells.

\section{Introduction}

Lipomas are common soft tissue neoplasms of mesenchymal origin; they involve the head and neck region in $20 \%$ of the cases, the oral cavity in 1 to $4 \%$ and other body regions in the remaining cases. A lipoma frequently originates in subcutaneous tissues and is delineated from the surrounding fatty tissue by a subtle fibrous capsule. The lesion can display different histological variants, such as fibrolipomas, intramuscolar lipomas, and spindle cell lipomas, and has an unknown pathogenesis with a median age of onset of 55 years. In physiologic condition adipose tissue contain adipocyte progenitor cells [1] that have the ability to differentiate along multiple lineage in vitro [2] and in vivo [3,4] and are alluded to as adipose-derived stem cells (ASCs) [5]. Human ASCs have been found to be $\mathrm{CD} 34+$, although they lean to be deprived of CD34 expression with culture time. CD34 is a marker for hematopoietic and mesenchymal stem cells (MSCs) and its presence is physiologically detectable in the perivascular environment of many tissues of post-natal organisms. The possibility that MSC derive from the vasculature can be explained with the maintenance of the stem cell pool [6] in a fast-recruitable compartment ready for tissue regen- 
eration [7].

The presence of $\mathrm{CD}_{3} 4^{+}$cells in lipomas is well-known [8] and is used to confirm the histological diagnosis $[9,10]$. A varying amount of $\mathrm{CD}^{2} 4^{+}$spindle cells is generally detectable within the lesion $[11,12]$ : they are more numerous near vessels [13], whereas the adipocytes and the other differentiated cells are negative for CD34 expression [14], leading to the hypothesis of an abnormal proliferation of normal CD34 perivascular cells in various types of lipomas and some liposarcomas [13]. A proliferation of CD34 ${ }^{+}$ dendritic cells in a variety of fibrovascular and fibrohistiocytic tumours has been reported [15] and hypothesized for Kaposi's sarcoma, also too [16]. As in human adipose tissues, a neoplastic transformation is thought to be related to the expression of a several number of genes [17]: cytogenetic abnormalities and differences in gene expression have been reported for lipomas when compared with normal adipose tissues, although no genes were solely expressed in lipomas or adipose tissues and overexpression of no known gene was identified in the lipomas [17].

Here, we present 20 cases of human lipomas, in different body regions. We performed analyses of primary cultures from the lipomas and challenged the cultures for CD34 at different stages, up to day 30, comparing these cultures with samples from normal adipose tissues. MSCs are characterized by a progressive loss of CD34 antigen in long term cultures in a terminal differentiation process: abnormalities in the physiological process of self-renewal of the stem cell pool could lead to abnormal proliferation observed in lipomas following tissue injury, as reported during anamnesis collection. The purpose of this study is to improve our understanding of cancer stem cells biology and to provide aid in the diagnosis of mesenchymal tumours through the identification of a key-role marker, such as CD34, supposedly involved in the pathogenesis of lipomas.

\section{Materials and Methods}

\section{Sample collection and cell culture}

After informed consensus, lipomas were collected from male and female patients in OUC of Plastic and Reconstructive Surgery. Surgical samples were gently removed and divided into 3 parts: the first part was used for histology, one for molecular biology and the second part was immersed in a digestive solution: penicillin 100U/ml/streptomycin $100 \mu \mathrm{g} / \mathrm{ml}, 0.6 \mathrm{ml}$ claritromycin $500 \mu \mathrm{g} / \mathrm{ml}$ in $4 \mathrm{ml}$ PBS $1 \mathrm{M}$, with 3 $\mathrm{mg} / \mathrm{ml}$ type I collagenase, $4 \mathrm{mg} / \mathrm{ml}$ dispase for $1 \mathrm{~h}$ at $37^{\circ} \mathrm{C}$. Once digested, the solution was filtered through $70 \mu \mathrm{m}$ Falcon strainers (Becton \& Dickinson, Franklin Lakes, USA). After filtration and washing, the pellet was resuspended in erythrocyte lysis buffer $(155 \mathrm{mM}$ ammonium chloride, $10 \mathrm{mM}$ potassium bicarbonate, 0,1 mM EDTA, pH 7.3) for $10 \mathrm{~min}$ at room temperature. The cell suspension was centrifuged at $1,300 \mathrm{rpm}$ for $7 \mathrm{~min}$ and the pellet resuspended in $5 \mathrm{ml}$ of Dulbecco's modified Eagle's medium (DMEM) with 10\% fetal bovine serum (FBS), $2 \mathrm{mM}$ L-glutammine, 100 $\mathrm{U} / \mathrm{ml}$ penicillin and $100 \mu \mathrm{g} / \mathrm{ml}$ streptomycin. Flasks were incubated at $37^{\circ} \mathrm{C}$ with $5 \% \mathrm{CO}_{2}$ and the medium changed twice a week. As controls, normal human adipose tissue samples were collected and managed as above.

\section{Flow cytometry and sorting}

Cells (from lipoma and normal adipose tissue) were detached using trypsin-EDTA (200 mg/L EDTA, $500 \mathrm{mg} / \mathrm{L}$ trypsin; Cambrex, Milan, Italy). Cells were incubated with primary antibody for $30 \mathrm{~min}$ at $4^{\circ} \mathrm{C}$, washed twice in PBS and incubated with a secondary antibody. Alternatively, cells were incubated directly with fluorescent-conjugated antibodies for $30 \mathrm{~min}$ at $4^{\circ} \mathrm{C}$, washed and resuspended in $0.6 \mathrm{ml}$ of PBS. Samples were analysed at day 0 (day of surgery) and day 30 by flow cytometry using a FACS Aria II (Becton\&Dickinson, Mountain View, CA). The antibodies used in this study were: anti-CD34 PE and anti-CD45 FITC (all purchased from Miltenyi-Biotech, Calderara di Reno, Bologna, Italy). CD34+ and CD34- cells were sorted for experiments. The purity of sorted population was routinely $90 \%$.

\section{Cell Proliferation assay}

The experimental group consisted of $\mathrm{CD}_{3} 4^{+}$cells from lipoma, while the control group consisted of $\mathrm{CD}^{+}{ }^{+}$cells from normal adipose tissue. The cell culture medium was exchanged every 2 days. Cell culture plates were obtained following 2, 4, 6, 8 and 10 -day culture periods. The entire medium was aspirated, prior to the addition of $1 \mathrm{ml}$ DMEM cell culture liquid and $200 \mu \mathrm{l}$ MTT ( $5 \mathrm{mg} / \mathrm{ml}$; Sigma, Milan, Italy) to each well and $5 \mathrm{~h}$ incubation at $37^{\circ} \mathrm{C}$. Following this, the supernatant was aspirated and $450 \mu \mathrm{l}$ DMSO (Sigma) was added to each well. The cells were then agitated for $15 \mathrm{~min}$, prior to $200 \mu \mathrm{l}$ of the liquid being removed and transferred to a 96-well plate. An enzyme-linked immunosorbent assay detector was used to monitor the photometric value at a wavelength of $570 \mathrm{~nm}$ (A570). The A570 was proportional to the rate of cell proliferation. At each time-point, seven wells were used to determine the mean and standard deviation values for the two groups (experimental and control).

Cell number was then determined from a standard curve (photometric value vs. cell number) generated by MTT extraction from counted cells. 


\section{Haematoxylin \& Eosin staining}

Samples (lipoma and normal adipose tissue) were fixed with $4 \%$ paraformaldehyde for $24 \mathrm{~h}$ at $40^{\circ} \mathrm{C}$, washed in PBS, incubated in $30 \%$ sucrose for 3 days, washed in PBS and then embedded in an optimal cutting temperature (OCT-purchased from Bio-Optica, Milan, Italy). They were then stored at $-80^{\circ} \mathrm{C}$ for hematoxylin and eosin (H\&E) staining. The frozen sections in the OCT were cut into $5 \mu \mathrm{m}$ thick slices, washed in distilled water for $10 \mathrm{~min}$. For H\&E staining, sections were placed in haematoxylin for 5 min. After three washes in distilled water, samples were placed in spring water for $20 \mathrm{~min}$. Then, the samples were placed in eosin acidified with acetic acid for $30 \mathrm{~s}$. They were then placed in $50 \%, 75 \%$, and 96\% EtOH and mounted with DPX.

\section{Immunofluorescence and Immunohistochem- istry}

- For Frozen sections in OCT, the samples were cut into $5 \mu \mathrm{m}$ thick slices, washed in distilled water for $10 \mathrm{~min}$. For immunofluorescence, tissue sections were incubated in PBS at 5\% milk for $60 \mathrm{~min}$ at room temperature. After a double washing in PBS for $10 \mathrm{~min}$ at room temperature, tissue sections were incubated overnight at $4^{\circ} \mathrm{C}$ with monoclonal anti-human antibodies (diluted 1:100 in PBS). Sections were washed in PBS three times for $10 \mathrm{~min}$ at room temperature and incubated for $90 \mathrm{~min}$ at $4^{\circ} \mathrm{C}$ with the secondary FITCor PE-conjugated antibody (diluted 1:200 in PBS; AbCam, Cambridge, UK).

Tissue sections incubated for $90 \mathrm{~min}$ at $4^{\circ} \mathrm{C}$ only with conjugated secondary antibodies were used as negative controls. The sections were then observed under a fluorescence microscope (Nikon Instruments Italia, Calenzano, Firenze, Italy). The antibodies used were: anti-CD34 (Miltenyi-Biotech).

- Immunohistochemical analyses were performed with a DAKO CYTOMATION kit (En Vision+System-HRP-AEC; Dako Italia, Milan, Italy) according to the manufacturer's protocol. The antibodies used were anti-adiponectin (AbCam) and an-
ti-PPAR-y (AbCam).

\section{Semi-quantitative analysis of cells}

In order to analyse the cellular characteristics of adipose cells present on lipoma and normal fatty tissue masked microscopic examinations were performed on immunostained sections. Briefly, two investigators analysed in a masked fashion at least 3 slides for each experiment by light microscopy using as the initial magnification. Each slide contained 3 sections of specimen and 5 fields of $322 \mu \mathrm{m}^{2}$ each were analysed for each tissue section. Experiments were performed at least three times.

\section{RT Profiler PCR array}

Total RNA from lipoma and normal human adipose tissue (control) samples were extracted with RNeasy Mini Kit (Qiagen Gmbh, Hilden, Germany), including DNase digestion with the RNase-Free DNase Set (Qiagen Gmbh). The RNA quality and concentration of the samples was measured using the NanoDropTM ND-1000 (Thermo Scientific). $500 \mathrm{ng}$ of total RNA of each sample were reverse transcribed with RT2 First Strand kit (Qiagen Sciences, Germantown, MD USA). Real-Time PCR was performed according to the user manual of the Human Mesenchymal Stem Cell RT2 Profiler PCR array (SABiosciences, Frederick, MD, USA) and of the Human Apoptosis $\mathrm{RT}^{2}$ Profiler PCR Array using $\mathrm{RT}^{2}$ SYBR Green ROX FAST Mastermix (SABiosciences). Human Mesenchymal Stem Cell RT2 Profiler PCR array profiles the expression of 84 genes involved in maintaining pluripotency and self-renewal status (Table 1) whereas Human Apoptosis RT2 ${ }^{2}$ Profiler PCR Array profiles the expression of 84 key genes involved in programmed cell death (Table 2). Thermal cycling and fluorescence detection were performed using a Rotor-Gene Q 100 (Qiagen). Data were analyzed using Excel-based PCR Array Data Analysis Templates (SABiosciences). Results are reported in term of ratio between the expression of each target gene in lipoma sample and in the control sample.

Table 1. Functional Gene Grouping related to Human Mesenchymal Stem Cell RT2 Profiler PCR array.

Stemness Markers:

FGF2 (bFGF), INS, LIF, POU5F1 (OCT4), SOX2, TERT, WNT3A, ZFP42.

MSC-Specific Markers:

ALCAM, ANPEP, BMP2, CASP3, CD44, ENG, ERBB2 (HER2), FUT4, FZD9, ITGA6, ITGAV, KDR, MCAM, NGFR, NT5E, PDGFRB, PROM1, THY1, VCAM1. Other Genes Associated with MSC:

ANXA5, BDNF, BGLAP (Osteocalcin), BMP7, COL1A1, CSF2 (GM-CSF), CSF3 (GCSF), CTNNB1, EGF, FUT1, GTF3A, HGF (Scatter Factor), ICAM1, IFNG, IGF1, IL10, IL1B, IL6, ITGB1, KITLG, MMP2, NES, NUDT6, PIGS, PTPRC, SLC17A5, TGFB3, TNF, VEGFA, VIM, VWF.

MSC Differentiation Markers:

Osteogenesis: BMP2, BMP6, FGF10, HDAC1, HNF1A, KDR, PTK2, RUNX2, SMURF1, SMURF2, TBX5.

Adipogenesis: PPARG, RHOA, RUNX2.

Chondrogenesis: ABCB1 (MDR1), BMP2, BMP4, BMP6, GDF5 (CDMP-1), GDF6, GDF7, HAT1, ITGAX, KAT2B, SOX9, TGFB1.

Myogenesis: ACTA2, JAG1, NOTCH1.

Tenogenesis: BMP2, GDF15 (PLAB), SMAD4, TGFB1. 
Table 2. Functional Gene Grouping related to Human Apoptosis $\mathrm{RT}^{2}$ Profiler PCR Array.

Induction of Apoptosis:

Death Domain Receptors: CRADD, FADD, TNF, TNFRSF10B (DR5).

DNA Damage: ABL1, CIDEA, CIDEB, TP53, TP73.

Extracellular Signals: CFLAR (CASPER), DAPK1, TNFRSF25 (DR3).

Other: BAD, BAK1, BAX, BCL10, BCL2L11, BID, BIK, BNIP3, BNIP3L, CASP1 (ICE), CASP10 (MCH4), CASP14, CASP2, CASP3, CASP4, CASP6, CASP8, CD27

(TNFRSF7), CD70 (TNFSF7), CYCS, DFFA, DIABLO (SMAC), FAS (TNFRSF6), FASLG (TNFSF6), GADD45A, HRK, LTA (TNFB), NOD1 (CARD4), PYCARD

(TMS1/ASC), TNFRSF10A, TNFRSF9, TNFSF10 (TRAIL), TNFSF8, TP53BP2, TRADD, TRAF3.

Anti-Apoptosis:

AKT1, BAG1, BAG3, BAX, BCL2, BCL2A1 (Bfl-1/A1), BCL2L1 (BCL-X), BCL2L10, BCL2L2, BFAR, BIRC3 (c-IAP1), BIRC5, BIRC6, BNIP2, BNIP3, BNIP3L, BRAF, CD27 (TNFRSF7), CD40LG (TNFSF5), CFLAR (CASPER), DAPK1, FAS (TNFRSF6), HRK, IGF1R, IL10, MCL1, NAIP (BIRC1), NFKB1, NOL3, RIPK2, TNF, XIAP (BIRC4).

Regulation of Apoptosis:

Negative Regulation: BAG1, BAG3, BCL10, BCL2, BCL2A1 (Bfl-1/A1), BCL2L1 (BCL-X), BCL2L10, BCL2L2, BFAR, BIRC2 (c-IAP2), BIRC3 (c-IAP1), BIRC6, BNIP2, BNIP3, BNIP3L, BRAF, CASP3, CD27 (TNFRSF7), CD40LG (TNFSF5), CFLAR (CASPER), CIDEA, DAPK1, DFFA, FAS (TNFRSF6), IGF1R, MCL1, NAIP (BIRC1), NOL3, TP53, TP73, XIAP (BIRC4).

Positive Regulation: ABL1, AKT1, BAD, BAK1, BAX, BCL2L11, BID, BIK, BNIP3, BNIP3L, CASP1 (ICE), CASP10 (MCH4), CASP14, CASP2, CASP4, CASP6, CASP8, CD40 (TNFRSF5), CD70 (TNFSF7), CIDEB, CRADD, FADD, FASLG (TNFSF6), HRK, LTA (TNFB), LTBR, NOD1 (CARD4), PYCARD (TMS1/ ASC), RIPK2, TNF, TNFRSF10A, TNFRSF10B (DR5), TNFRSF25 (DR3), TNFRSF9, TNFSF10 (TRAIL), TNFSF8, TP53, TP53BP2, TRADD, TRAF2, TRAF3.

DEATH Domain Proteins: CRADD, DAPK1, FADD, TNFRSF10A, TNFRSF10B (DR5), TNFRSF11B, TNFRSF1A, TNFRSF1B, TNFRSF21, TNFRSF25 (DR3),

TRADD.

Caspases and Regulators:

Caspases: CASP1 (ICE), CASP10 (MCH4), CASP14, CASP2, CASP3, CASP4, CASP5, CASP6, CASP7, CASP8, CASP9, CFLAR (CASPER), CRADD, PYCARD (TMS1/ASC).

Caspase Activators: AIFM1 (PDCD8), APAF1, BAX, BCL2L10, CASP1 (ICE), CASP9, NOD1 (CARD4), PYCARD (TMS1/ASC), TNFRSF10A, TNFRSF10B (DR5), TP53.

Caspase inhibitors: CD27 (TNFRSF7), XIAP (BIRC4).

\section{Statistical analysis}

Statistical analysis was performed using SPSS version 13.0 statistical software (SPSS, Inc., Chicago, IL, USA) and group comparisons were conducted using a t-test. All values are presented as the mean \pm standard error (SE). $\mathrm{P}<0.05$ was considered to indicate a statistically significant difference.

\section{Results}

After informed consensus, we performed surgery on male and female patients affected by lipomas: lipomas were collected from the trunk, from the leg, from the chest and from the neck. All patients were in good health and with normal BMI.

Cytometric analyses, performed after digestion of surgical samples, showed that CD34 expression was $18,42 \pm 2.2 \%$ of total cell population from lipoma; CD45 positive cells were $3,02 \pm 1 \%$ and all were positive also CD34 marker (Fig. 1A). In normal tissues CD34 positive cells were $22,98 \% \pm 3.4 \%$ of total cell population and CD45 was $0,03 \pm 1 \%$ and, also in this case, all were positive for CD34 marker (Fig. 1B). We sorted $\mathrm{CD} 34^{+}$cells both in normal and in pathological samples and we used these cells to continue the experiment. We also cultured non-sorted population to prospectively compare the expression of CD34 (data not shown).

After 30 days of culture, we analysed CD34 and CD45 expression in sorted and non sorted cell cultures. Interestingly, we found that in normal non sorted cultures, CD34 expression was very lower than at day 0 ; this event did not occur in pathological cultures, where the expression remained more or less at the same levels of day 0 (Fig. 1C,D).

The MTT assay's results unveiled that the CD34+ cells from the lipoma were capable of a faster growth and proliferation compared to CD34+ ASCs. At the second and fourth-day time-points, a noteworthy dissimilarity was witnessed between the control and experimental groups, and the cell proliferation of the experimental group was higher than that of the control group; significant differences between the two groups were also identified at the sixth, eighth and tenth-day time-points (Fig. 2).

Histopathology sections revealed (table 3) the absence of ruptured adipocytes in both lipoma and normal fatty tissue. Adipocytes differing in size have been found: the portion of small adipocytes was critically higher in lipoma tissue than in normal adipose tissue (Fig. 3A,C). CD34 was present in both lipoma and normal adipose tissue. Moreover, it has been repeatedly detected that the CD34 positivity was more prevalent round small adipocytes in lipoma tissue (Fig. 3B,D).

Adiponectin expression was lower in CD34+ lipoma cells contrasted with normal $\mathrm{CD} 34^{+}$ASCs in all patients (Fig. 4A,B). However, in our specimens no noticeable difference in the expression of PPAR- $\gamma$ was ascertained between $\mathrm{CD} 34^{+}$lipoma cells and normal CD34+ ASCs (Fig. 4C,D). 
Table 3. Cells were scored from not present (-) to abundantly present (+++)

\begin{tabular}{|c|c|c|c|c|c|}
\hline & ruptured adipocytes & $\begin{array}{l}\text { small adipo- } \\
\text { cytes }\end{array}$ & CD34 positive cell & CD34+small size & CD34+big size \\
\hline normal fatty tissue. & - & ++ & ++ & ++ & +++ \\
\hline lipoma fatty tissue. & - & +++ & ++ & +++ & ++ \\
\hline
\end{tabular}

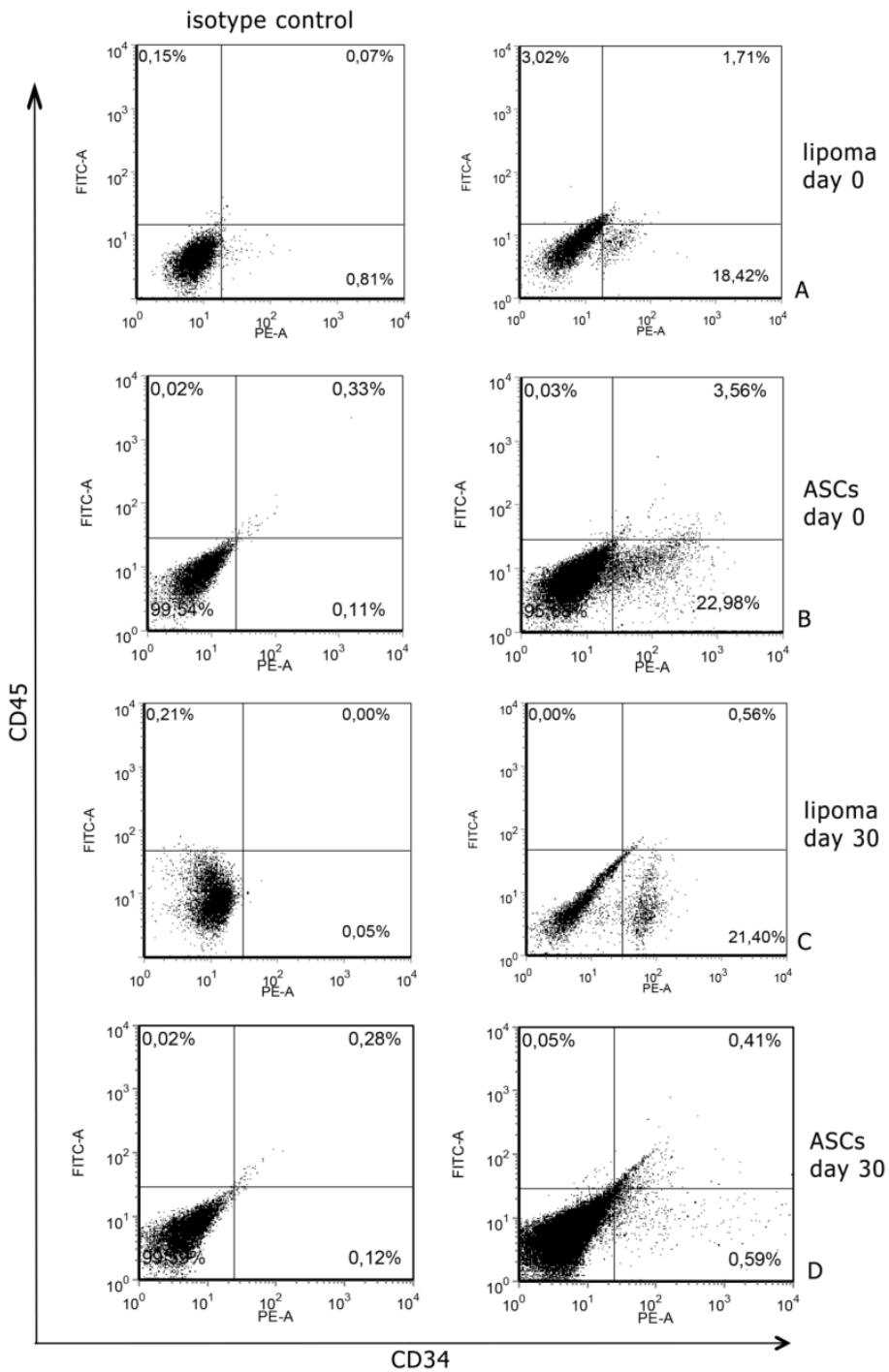

Fig. 1: Representative flow cytometry analysis performed at day $\mathbf{0}$ and $\mathbf{3 0}$ of culture. A. A significant number of lipoma cells at day 0 are clearly positive for CD34; B. A significant number of ASCs at day 0 are clearly positive for CD34+. C. After 30 days of culture, CD34 levels, in lipoma cells, remained at the same levels of day 0; D. After 30 days of culture, CD34 levels, in ASCs, significantly decreased.

Growth Curve

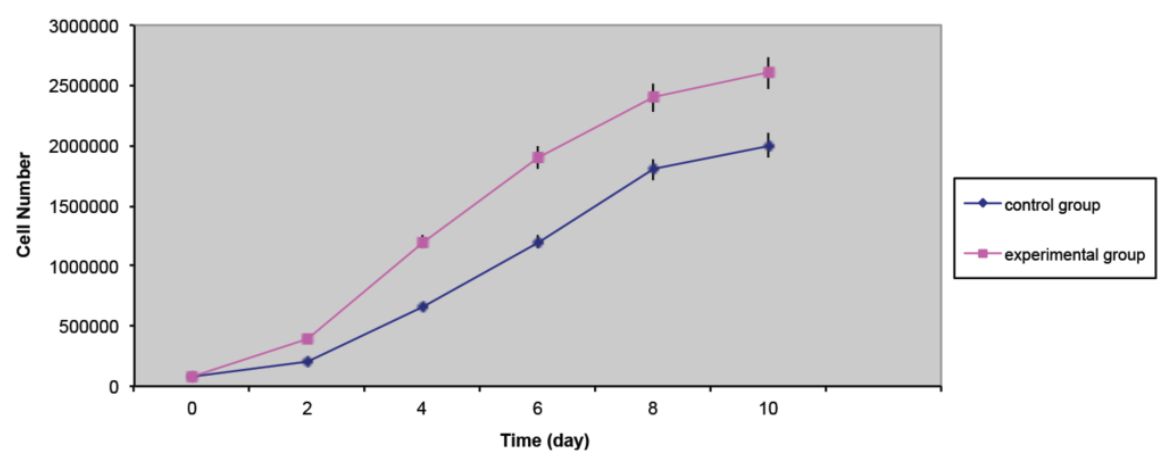

Fig. 2: Representative image showing growth curves of CD34+ lipoma cells (experimental group) compared to CD34+ adipose cells (control group) displaying a different mean doubling time. $P$ value $<0.05$. 

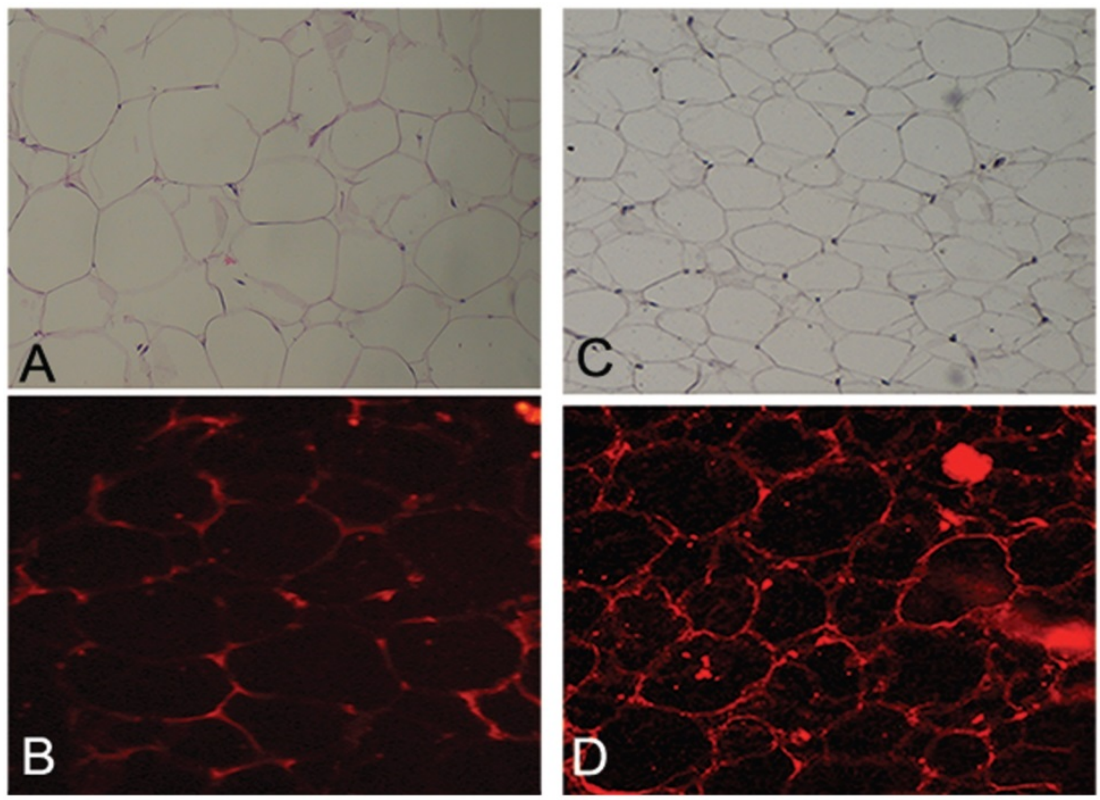

Fig. 3: Figure showing morphological assessment of adipocytes: (A) H\&E staining displaying normal morphology of adipocytes in normal tissue appearing identical in shape and volume (Original Magnification X100); (B) Immunofluorescence staining for CD34 displaying, in normal adipose tissue, uniform and unrepresentative expression (Original magnification X200); (C) H\&E staining displaying, in lipoma tissue, a different morphology of adipocytes. They appear different in shape and volume with many small adipocytes (Original Magnification X100); (D) Immunofluorescence staining for CD34 displaying, in lipoma adipose tissue, an ubiquitous non-uniform expression of small adipocytes (Original magnification X200).
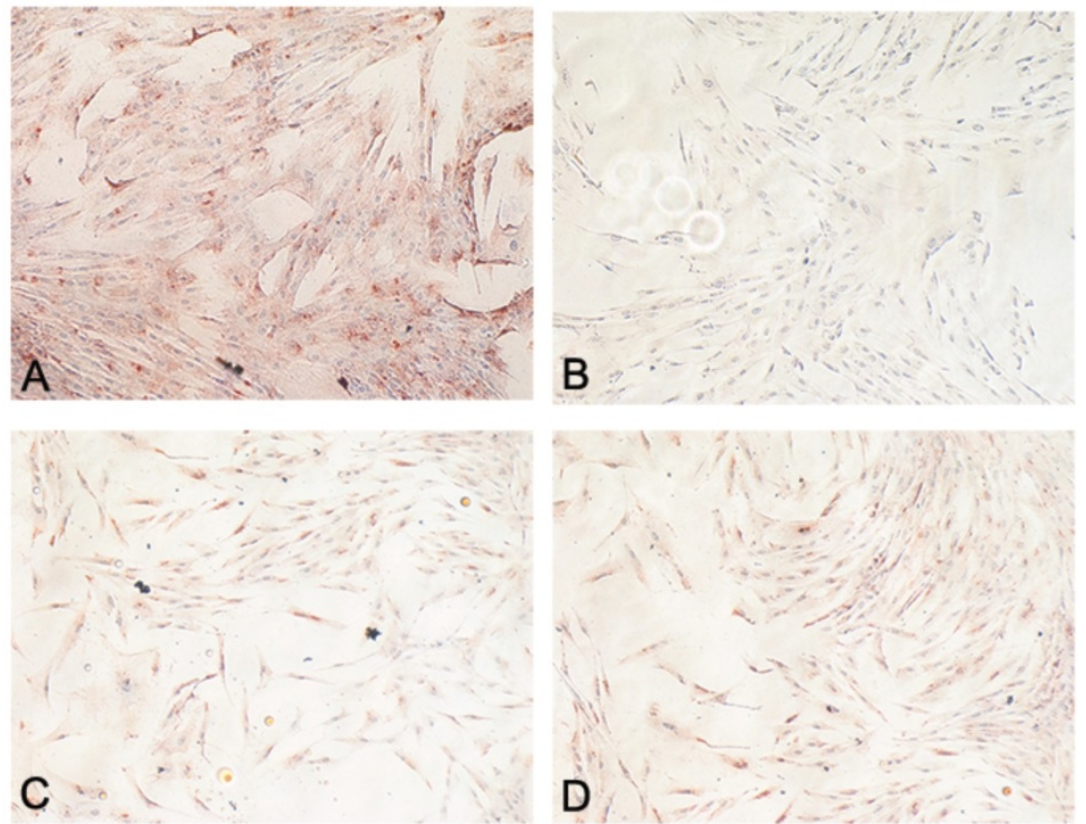

Fig. 4: Figure showing functional assessment of adipocytes: (A) Immunohistochemistry staining for adiponectin in CD34+ ASCs (Original magnification X200); (B) Immunohistochemistry staining for adiponectin in CD34+ lipoma cells (Original magnification X200); (C) Immunohistochemistry staining for PPAR-Y in CD34+ ASCs (Original magnification X200); (D) Immunohistochemistry staining for PPAR-Y in CD34+ lipoma cells (Original magnification X200).

In order to characterize the stemness properties of the stem cells present in lipoma's tissue, the gene expression for stemness markers has been performed and results are shown in figure 5A. In the figure is it possible to note an up regulation for: CD54, CD34, CD90, CD44, Nanog, Oct3/4, IL6, TNF alfa, leptin, Adiponectin, Restin and PPARY. Moreover he have found interesting up regulation of several genes, that we have grouped (Fig 5B-H) for specific activity. In detail we have dectected gene expression alteration on: Bone morphogenetic protein 6 (BMP6), Colony stimulating factor 2 (granulocyte-macrophage) (CSF2), Catenin (cadherin-associated protein), (CTNNB1), Fibroblast growth factor 10 (FGF10);Interferon, gamma (IFNG), Insulin-like growth factor 1 (somatomedin C) (IGF1), Insulin (INS), Integrin, alpha $X$ (complement component 3 receptor 4 subunit)(ITGAX),Notch 1 (NOTCH1), 
Nudix (nucleoside diphosphate linked moiety $X)$-type motif 6(NUDT6), POU class 5 homeobox 1 (POU5F1); Peroxisome proliferator-activated receptor gamma (PPARG);Protein tyrosine phosphatase, receptor type, C (PTPRC);Solute carrier family 17 (anion/sugar transporter), member 5 (SLC17A5); SMAD family member 4 (SMAD4); SRY (sex determining region Y)-box 9 (SOX9), Tumor necrosis factor (TNF); Vascular cell adhesion molecule 1 (VCAM1);Von Willebrand factor (VWF); Wingless-type MMTV integration site family, member $3^{\circ}$ (WNT3A), SRY (sex determining region $\mathrm{Y}$ )-box 2 (SOX2), Telomerase reverse transcriptase (TERT); Wingless-type MMTV integration site family, member $3^{\circ}$ (WNT3A);Melanoma cell adhesion molecule (MCAM), Nerve growth factor receptor (NGFR), Vascular cell adhesion molecule 1 (VCAM1),Colony stimulating factor 2 (granulocyte-macrophage) (CSF2),Interferon, gamma (IFNG), Insulin-like growth factor 1 (somatomedin C) (IGF1), Interleukin 6 (interferon, beta 2) (IL6), Protein tyrosine phosphatase, receptor type, C (PTPRC), Fibroblast growth factor 10 (FGF10), Peroxisome proliferator-activated receptor gamma (PPARG).

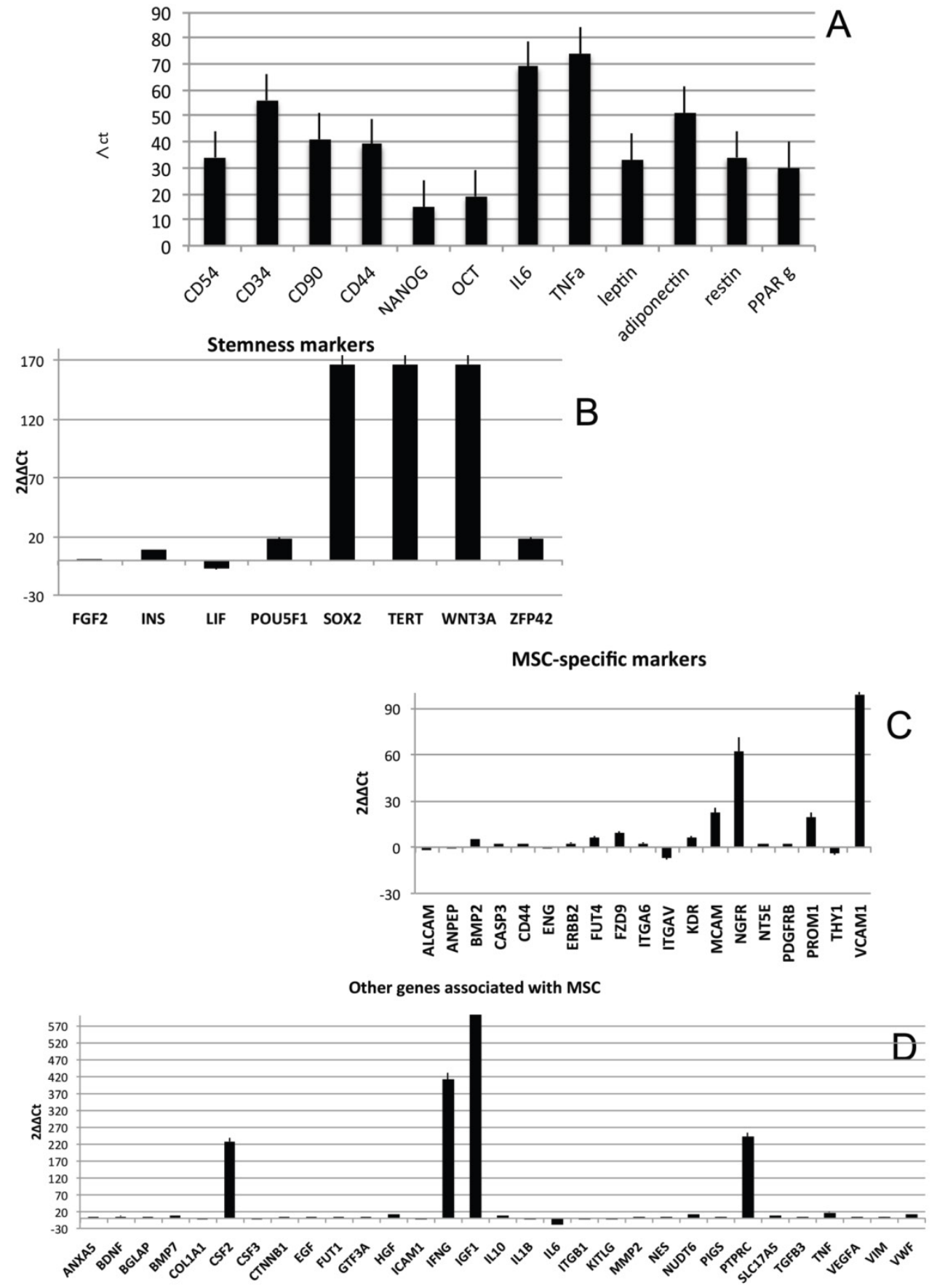


E

Osteogenesis
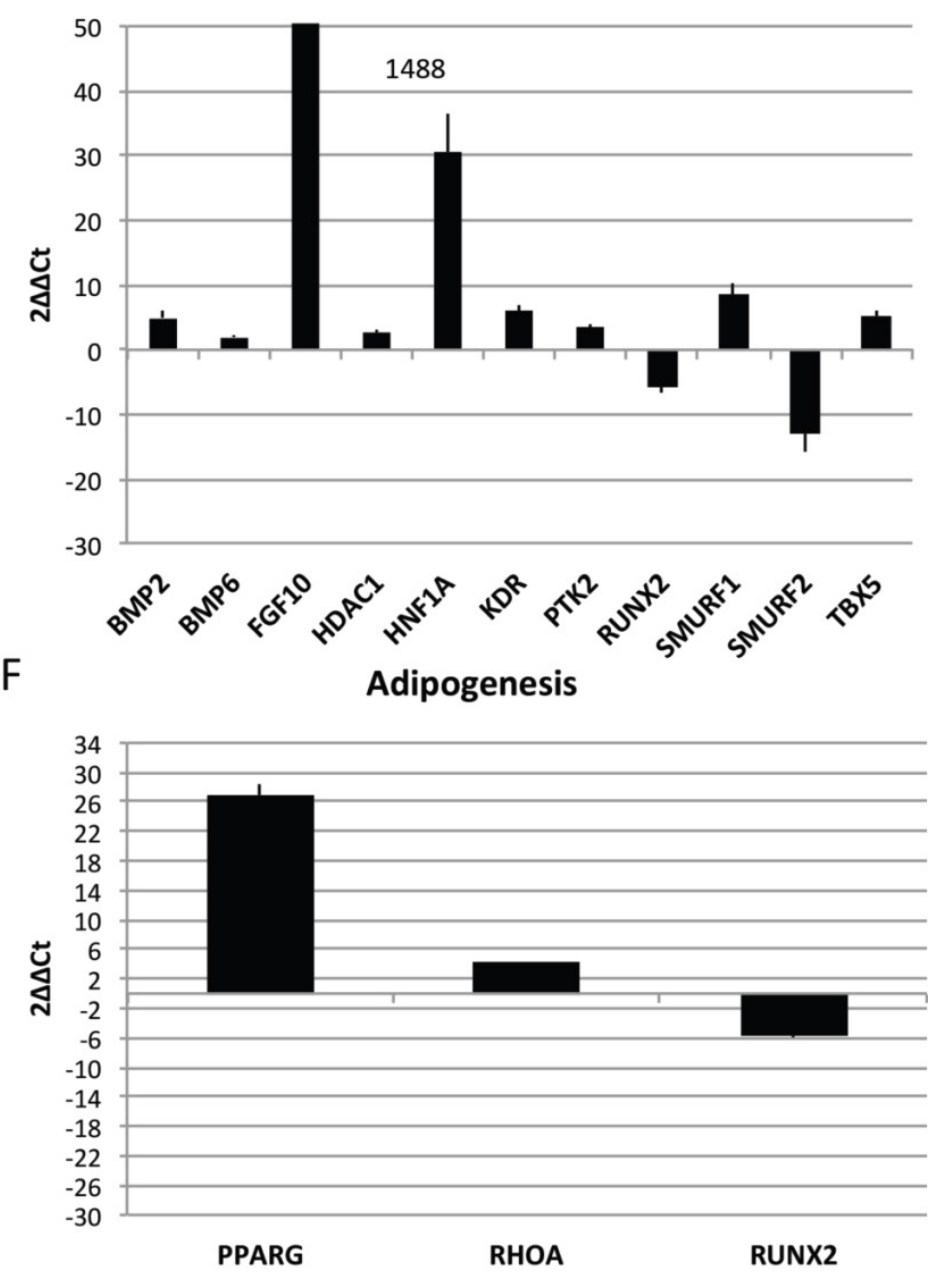

G Chondrogenesis

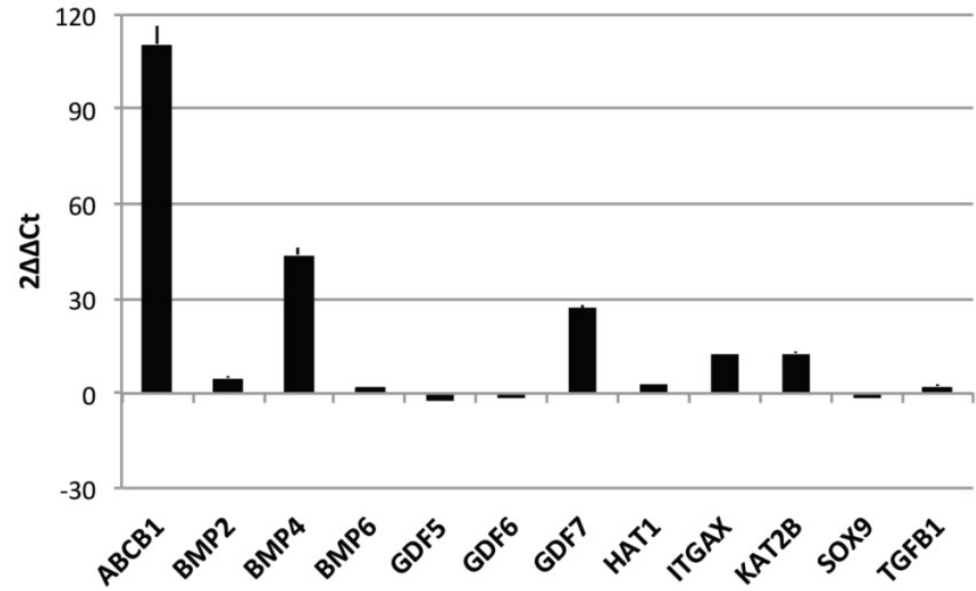


H

Tenogenesis

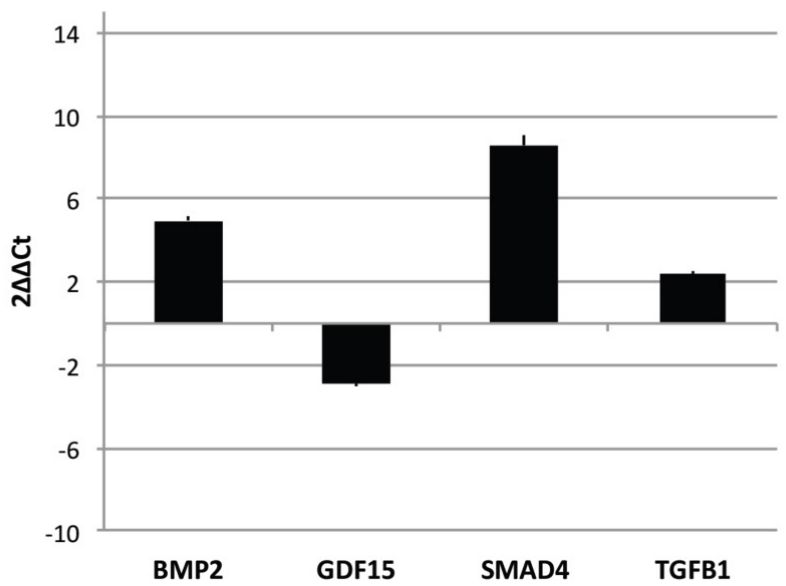

Negative regulation of apoptosis

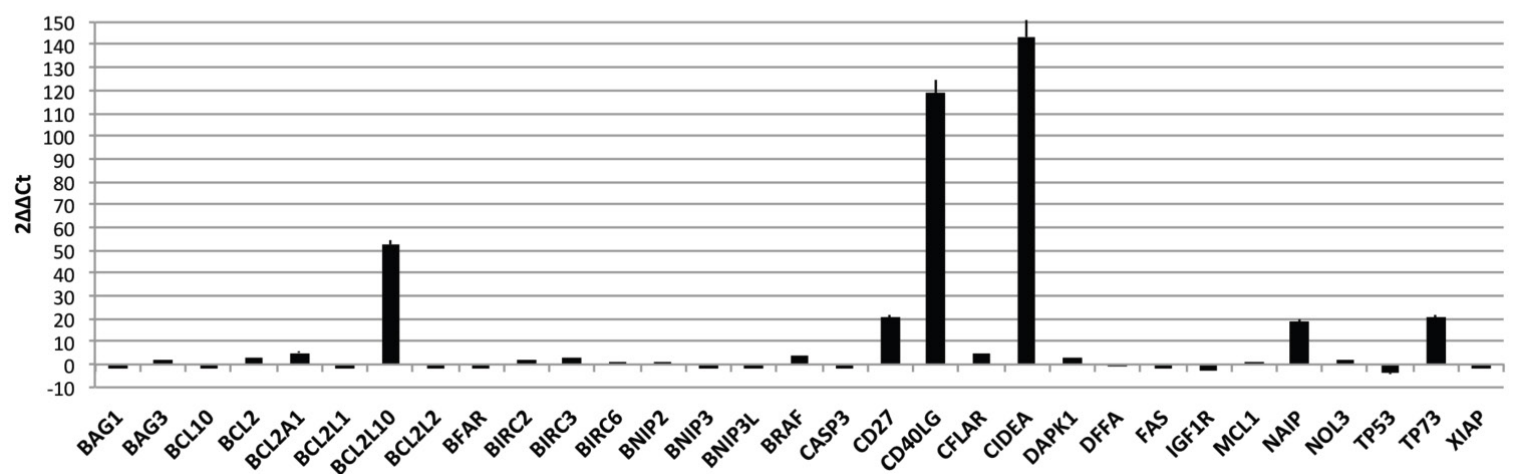

Positive regulation of apoptosis

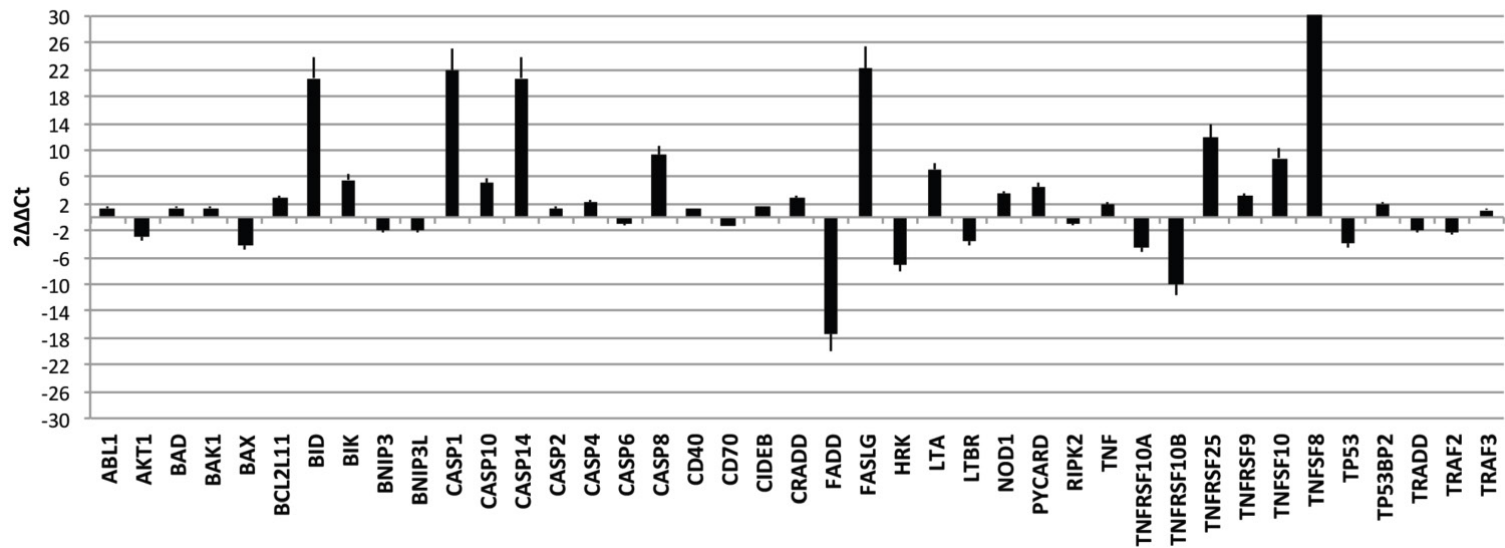



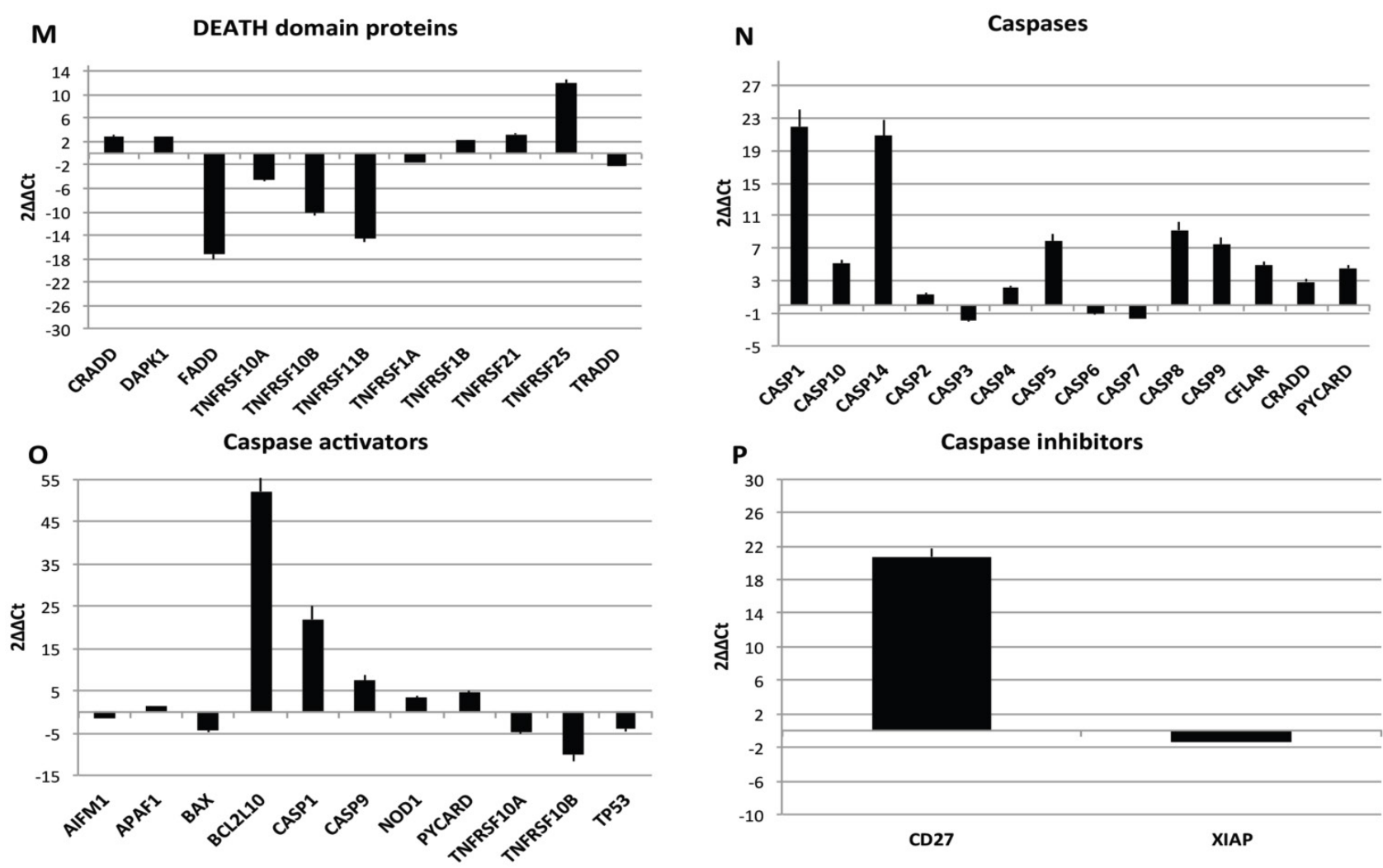

Fig. 5: Transcriptional profiles of lipoma and normal adipose tissue. (A) Gene expression related to superficial markers such as CD 54,34,90,44; inflammatory related markers such as IL6 and TNFa; adipogenic markers such as leptin, adiponectin, restin PPARg; and trascriptrional factors such as NANOG and OCT; (B) Gene expression related to stemness specific markers; (C, D) Gene expression related to mesenchymal lineage specific markers; (E) Gene expression related to osteogenesis specific markers; (F) Gene expression related to adipogenesis specific markers; (G) Gene expression related to chondrogenesis specific markers; (H) Gene expression related to tenogenesis specific markers; (I, L) Gene expression related to negative regulation of apoptosis and to positive regulation; (M) Gene expression related to DEATH domain protein; $(\mathbf{N}, \mathbf{O}, \mathbf{P})$ to caspase related way: caspase protein $(\mathrm{N})$, caspase activator $(\mathrm{O})$ capsase inhibitor (P).

By contrast a down regulation for only 3 genes is reported: Growth differentiation factor 6 (GDF6), Colony stimulating factor 3 (granulocyte) (CSF3), Bone morphogenetic protein 4 (BMP4)

To great interest is the higher expression of gene related to vascularization process are present such as VCAM1, VEGFA and VWF and, notably, also higher expression of stemness related markers by mesenchymal stem cells such as TERT (direct to protect telomeres) and WNT3 involved on stem cell niche and ZFP42 characterizing pluripotent stem cells. These markers indicate that CD34+ lipoma cells show higher vasculogenic properties and a bigger resistance in aging.

Finally, we centered our attention on apoptotic pathway gene expression (Fig. 5I-P), where is reported an up regulation for BCL2-like 10 (apoptosis facilitator) (BCL2L10), BH3 interacting domain death agonist (BID), Caspase 1, apoptosis-related cysteine peptidase (interleukin 1, beta, convertase) (CASP1), Caspase 14, apoptosis-related cysteine peptidase (CASP14), CD27 molecule (CD27), CD40 ligand (CD40LG), Cell death-inducing DFFA-like effector a (CIDEA), Fas ligand (TNF superfamily, member 6)
(FASLG), NLR family, apoptosis inhibitory protein (NAIP),Tumor necrosis factor (ligand) superfamily, member 8 (TNFSF8), Tumor protein p73 (TP73), Cell death-inducing DFFA-like effector a(CIDEA), Tumor protein p73 (TP73); Tumor necrosis factor receptor superfamily, member 25 (TNFRSF25), BCL2-like 1(apoptosis facilitator) (BCL2L10), BH3 interacting domain death agonist(BID), Caspase 1, apoptosis-related cysteine peptidase (interleukin 1, beta, convertase) (CASP1), Caspase 14, apoptosis-related cysteine peptidase (CASP14), CD27 molecule (CD27),Fas ligand (TNF superfamily, member 6) (FASLG), BCL2-like 10 (apoptosis facilitator) (BCL2L10); CD40 ligand (CD40LG); BCL2-like 10 (apoptosis facilitator) (BCL2L10). By contrary a down regulation has been observed on expression related to Fas (TNFRSF6)-associated via death domain (FADD);F as (TNFRSF6)-associated via death domain (FADD), Tumor necrosis factor receptor superfamily, member 10b (TNFRSF10B); Tumor necrosis factor receptor superfamily, member 11b (TNFRSF11B).

Resuming, in lipoma's tissue we found a greater expression of several genes associated to the increase of negative regulator of apoptotic event and a down- 
regulation of apoptotic markers.

\section{Discussion}

Histolopathological classification and classification of tumours of the soft (mesenchymal) tissues includes Tumours of fibrous tissue (such as fibroma, fibrosarcoma, others sarcoid), Tumours of fat tissue (lipoma and liposarcoma), Tumours of muscle tissue (such as leiomyoma, leiomyosarcoma, rhabdomyoma, rhabdomyosarcoma), Tumours of blood and lymph vessels (such as haemangioma, angiosarcoma, glomus tumour, lymphangioma, lymphangiosarcoma), mesenchymal tumours of peripheral nerves and mast cell tumour (mastocytoma) [18].

The simple lipoma is the most common, accounting for $80 \%$ of adipose tissue tumours. The angiolipoma, the lipoblastoma and intermuscular lipoma constitute the 18\% [19]. Cytogenetic abnormalities have been reported: rearrangement of the $12 q 1415$, the $6 p$ and $13 q$ deletion [20]. The lipoma appears in the third decade of life, when the fatty tissue begins to accumulate. Diabetes and obesity are conditions favourable to its development. Histologically, lipoma is composed of mature fat cells, circumscribed by a thin fibrous capsule. It can be the site of traumatic bleeding or necrosis with the presence of granulomas [21]. Malignant adipose tissue tumors are also known as liposarcomas [22]. Liposarcomas occur in patients of all ages, most frequently in the sixth decade, affecting mainly the thigh, the popliteal area and the retroperitoneal fat. We recognize different histological variants with hypochromatic, ovoid or round nuclei and mitotic figures.

Histology and imaging are two completing morphological techniques playing a pivotal role in the diagnosis and management of soft tissue tumors. Imaging enables to identify benign lesions like lipoma. There is no strict canon for diagnosis of a sarcomas basing on radiological findings, however, in the presence of necrosis and invasion, malignancy can strongly be suspected. Furthermore, these benign and malignant lesions may be confused/misunderstood. At times, even the most experienced pathologists strive to draw up an accurate classification of lipomatous tumours. Given that the diverse types of lipomatous tumours display significantly different biological behaviours, a correct diagnosis is fundamental. Lipoma-derived stem cells lines are a treasured model system for studying the genesis of liposarcomas and for preclinical inquiries. Poorly differentiated and aggressive neoplasms have been correlated to the expression of the embryonic stem cell markers OCT3/4 and NANOG [23]. In our case, the expression of embryonic markers is low enough to justify the lack of aggressiveness and high differentiation of the tu- mour. The expression of cell surface antigens is frequently employed as cancer stem cells (CSC) markers. Lipoma-derived stem cells may originate from hASCs and we ascertained the expression of CD34, CD90, CD44 and CD54, all expressed on hASCs $[2,3,5]$. The CD90 exhibition has not been related to sarcoma CSCs in contrast to CD133 marker. The low expression of CD90 in lipoma samples shows the different histologic nature of lipoma compared to liposarcoma. CD44 is a CSC marker in many neoplasms albeit it has not been employed as sarcoma CSC marker [24]. Nevertheless, CD44, as well as CD54, is expressed in lipoma cell lines. Their presence can be explained by the ability of local tumour progression and the rich neo-angiogenesis. Interestingly, lipoma increase in size with body weight gain, but weight loss does not decrease their size, this behaviour can be justified by the fact that lipoma cells may originate from mesenchymal stem cells [25]. In adipose tissue, CD34 ${ }^{+}$cells appear extensively distributed and form a well-organized network that surrounds lobes and lobules, intimately associated with microvessels and adipocytes. Our results showed small adipocytes surrounded by $\mathrm{CD} 34^{+}$cells in lipoma enhancing adipogenesis and angiogenesis, respect to fatty tissue. Accordingly, in normal white adipose tissue, various authors have identified the immunophenotype of different components of stromal vascular fraction (SVF) $[4,5,26,27]$ based on CD34 cells positivity: preadipocytes, mature endothelial cells, and pericytes. Moreover, the immunophenotype differences, respect to lipoma tissue, include loss of CD34 expression with cell expansion with a concomitant increase of differentiative markers demonstrating adipogenic, osteogenic, and chondrogenic characteristics. In lipoma tissue, the persistence of CD34 positivity is associated with increased replicative capacity and maintenance of cell immaturity. Their mesenchymal stem cell properties play an important role in fibrosis and tumor stroma formation, due to immunological tolerance (immunomodulation) and to capability of synthesize and remodel the extracellular matrix. Consequently, CD34+ cells are a regulator of stromal collagen content and contribute to activate local fibroblasts through increased proliferative capacity (originating new fibroblasts), stimulation of growth factors and extracellular matrix protein. Moreover, CD34+ cells secrete VEGF, PDGF and IL-8, which promote angiogenesis $[28,29]$ and support postnatal neovascularization $[5,30]$.

CD34+ cells, in lipoma, participate in tumor stroma formation and progression (in this case, also alphaSMA+). In lipoma, these cells are present in all or most cells, thereby contributing significantly to diagnosis. In other soft tissue tumours, the 
co-expression of other markers is common and this also has the meaning of malignancy.

Moreover, the enhanced proliferation activity in lipomas suggested that the augmentation of lipoma tissue may be due to the increased adipocyte turnover. Interestingly, our study revealed some characteristics associated with obesity. Similar to obesity, lipoma-derived adipocytes exhibited adiponectin downregulation and leptin upregulation. This suggested an adipocyte dysfunction. Moreover, in lipoma tissue, a terminal differentiation of lipoblasts was observed. The lipoma tissue revealed to be composed of mature adipocytes due to the PPAR-y expression that was unchanged between lipoma and normal fatty tissue.

Inflammatory response is a customary and valuable basic pathological process. Very often, obese patients have elevated levels of C-reactive protein (CRP) due to a pro-inflammatory effect produced by some cytokines such as IL-6. In the microenvironment of inflammation, mediators can play a precise regulatory role in the mesenchymal stem cells' activity and they have a function in the damaged tissues. In our study we suggest that the effects of the CD34's persistent positivity are partially justified by the fact that injured tissues send signals to the cells, that are activated and exhibit an anti-inflammatory protein, tumour necrosis factor- (TNF) alpha, and a pro-inflammatory protein, interleukin-6. As it is well known apoptosis is proven to be a mechanism of defence against cancer. Albeit cells can commit suicide through various ways, the majority of cell death is arbitrated by the mitochondrial (intrinsic) pathway that is started by a profusion of signals, such as developmental cues, growth factor deprivation and DNA damage, in addition to several standard anti-cancer treatments. In this context, members of the BCL-2 family are crucial moderators of apoptotic cell death both in health and disease and their deregulation has been shown in cancer. Thereby, apoptosis might lead to genomic instability during phases of proliferation by promoting the rise of pathologic clones and consequent replication of stress-associated DNA damage. The pro- and anti-apoptotic BCL-2 family proteins intimately interact at the mitochondrial membrane and moderate the pathway of intrinsic apoptosis. Customary cytotoxic agents hinder upstream signaling pathways converging at the BCL-2 family level. Both exogenous and (epi)genetic alterations/modifications in the wide series of elements modulating apoptosis, result in progressive/advancing counteraction of pro-death signals. Extrapolating to human tumours, the capability of apoptotic cells to actively enhance proliferation of surrounding cells, for example by secreting mitogens, might be of major significance. More recently, studies have hinted that caspases, that are proapoptotic proteins, can replace dying cells by stimulating the proliferation of contiguous surviving cells. Clearly, proliferation induced by apoptosis can be crucial for the initiation and development of tumours. Nonetheless, apoptotic cells may affect surrounding surviving cells in other manners. We here can hypothesize that cell-cell interaction probably/possibly links proliferation and cell death either actively through signals evoked from apoptotic cells, or passively. Furthermore, immune and inflammatory cells supply an additional level of communication between dying and proliferating cells. Dying cells activate neutrophils, macrophages, mast cells and dendritic cells that secrete mitogenic cytokines like IL1, IL6 or TNFa. Hence, the immune and inflammatory systems, besides immune surveillance that is their major function, also promote malignant transformation under certain circumstances. Briefly, we suggest that comprehending the influence of death-driven proliferation on tumorigenesis, either mediated by inflammatory signals or directly, can uncover a new way to ameliorate therapy and possibly intercept the development of cancer.

These results suggest that other mechanisms are involved in increased activity of lipoma dependent on genetic or micro environmental factors [31]. However further studies will be needed in order to explicate the detailed processes concealed under this phenomenon.

The potential roles of the CD34 family include increasing proliferation (replicative capacity) and blocking differentiation of stem or progenitor cells that therefore maintain there undifferentiated progenitor/stem-cell phenotype (immaturity or stemness of the cell). Moreover, CD34 ${ }^{+}$plays an important role in wound healing, tissue repair, fibrosis and in tumour stroma formation. The CD34-positive lipoblasts are thought to depict an uncommitted cell able to differentiate multidirectionally in a mesenchymal way, and this hints the presence of an inverse correlation between CD34 expression and differentiation. Presence of $\mathrm{CD}_{3} 4^{+}$stromal cells is constantly noticeable in the stroma of lipoma. In conclusion, the presence of these cells may play some role in regulating the growth or the differentiation of tumour cells displaying a nodule appearance such as lipoma.

\section{Acknowledgements}

We thank Prof. Giuseppina Caraglia B.A., English language expert and assistant for Department of Sciences and Environmental, Biological, Pharmaceutical Technologies, Caserta, Second University of Naples for providing excellent technical revision and support. 


\section{Competing Interests}

The authors have declared that no competing interest exists.

\section{References}

1. Casadei A, Epis R, Ferroni L et al. Adipose tissue regeneration: a state of the art. J Biomed Biotechnol. 2012; 2012: 462543.

2. Nicoletti GF, De Francesco F, D'Andrea F, et al. Methods and procedures in adipose stem cells: state of the art and perspective for translation medicine. J Cell Physiol. 2015; 14: 233-42.

3. Ferraro GA, De Francesco F, Nicoletti G, et al. Human adipose CD34+CD90+ stem cells and collagen scaffold constructs grafted in vivo fabricate loose connective and adipose tissue. J Cell Biochem. 2013; 114: 1039-49.

4. Desiderio V, De Francesco F, Schiraldi C, et al. Human $\mathrm{Ng}_{2}+$ adipose stem cells loaded in vivo on a new crosslinked hyaluronic acid-Lys scaffold fabricate a skeletal muscle tissue. J Cell Physiol. 2013; 228: 1762-73.

5. De Francesco F, Tirino V, Desiderio V, et al. Human CD34/CD90 ASCs are capable of growing as sphere clusters, producing high levels of VEGF and forming capillaries. PLoS One. 2009; 4: 66537.

6. Watt FM, Hogan BL. Out of Eden: stem cells and their niches. Science. 2000; 287: $1427-30$.

7. Meirelles Lsa S, Fontes AM, Cvas DT, et al. Mechanisms involved in the therapeutic properties of mesenchymal stem cells. Cytokine Growth Factor Rev. 2009; 20: 419-27.

8. Templeton SF, Solomon AR Jr. Spindle cell lipoma is strongly CD34 positive. An immunohistochemical study. J Cutan Pathol. 1996; 23: 546-50.

9. Coimbra F, Lopes JM, Figueiral H, et al. Spindle cell lipoma of the floor of the mouth. A case report. Med Oral Patol Oral Cir Bucal. 2006; 11: E401-3.

10. Cheah A, Billings S, Goldblum J, et al. Spindle cell/Pleomorphic lipomas of the face: an under-recognized diagnosis. Histopathology. 2015; 66: 430-7.

11. Kaku N, Kashima K, Daa T, et al. Multiple spindle cell lipomas of the tongue: report of a case. APMIS. 2003; 111: 581-5.

12. Usta U, Turkmen E, Mizrak B, et al. Spindle cell lipoma in an intramuscular lipoma. Pathol Int. 2004; 54: 734-9.

13. Silverman JS, Tamsen A. Fibrohistiocytic differentiation in subcutaneous fatty tumors. Study of spindle cell, pleomorphic, myxoid and atypical lipoma and dedifferentiated liposarcoma cases composed in part of CD34+ fibroblasts and FXIIIa+ histiocytes. J Cutan Pathol. 1997; 24: 484-93.

14. Billings SD, Henley JD, Summerlin DJ, et al. Spindle cell lipoma of the oral cavity. Am J Dermatopathol. 2006; 28: 28-31.

15. Val-Bernal JF, Martino M, Garces CM, et al. Soft-tissue angiomatosis in adulthood: a case in the forearm showing a prominent myxoid adipose tissue component mimicking liposarcoma. Pathol Int. 2005; 55: 155-9.

16. Gurzu S, Ciortea D, Munteanu R, et al. Mesenchymal-to-endothelial transition in Kaposi sarcoma: a histogenetic hypothesis based on a case series and literature review. PLoS One. 2013; 8: e71530.

17. Skubitz KM, Cheng EY, Clohisy DR, et al. Differential gene expression in liposarcoma, lipoma and adipose tissue. Cancer Invest. 2005; 23: 105-18.

18. Van Unnik JA. Classification and grading of soft-tissue sarcomas. Hematol Oncol Clin North Am. 1995; 9: 677-700.

19. Bancroft LW, Kransdorf MJ, Peterson JJ et al. Benign fatty tumors: classification, clinical course, imaging appearance and treatment. Skeletal Radiol. 2006; 35: 719-33.

20. Rubin BP, Fletcher CD. The cytogenetics of lipomatous tumours. Histopathology. 1997; 30: 507-11.

21. Drevelegas A, Pilavaki M, Chourmouzi D. Lipomatous tumors of soft tissue: MR appearance with histological correlation. Eur J Radiol. 2004; 50: 257-67.

22. Dodd LG. Update on liposarcoma: a review for cytopathologists. Diagn Cytopathol. 2012; 40: 1122-31.

23. Mimeault M, Batra SK. Molecular biomarkers of cancer stem/progenitor cells associated with progression, metastases, and treatment resistance of aggressive cancers. Cancer Epidemiol Biomarkers Prev. 2014; 23: 234-54.

24. Camerlingo R, Ferraro GA, De Francesco $F$, et al. The role of CD44+/CD24-/low biomarker for screening, diagnosis and monitoring of breast cancer. Oncol Rep. 2014; 31: 1127-32.

25. Lin TM, Chang HW, Wang KH, et al. Isolation and identification of mesenchymal stem cells from human lipoma tissue. Biochem Biophys Res Commun. 2007; 361: 883-9.

26. Zuk PA, Zhu M, Ashjian P, et al. Human adipose tissue is a source of multipotent stem cells. Mol Biol Cell. 2002; 13: 4279-95.

27. Gimble JM, Katz AJ, Bunnell BA. Adipose-derived stem cells for regenerative medicine. Circ Res. 2007; 100: 1249-60.

28. Diaz-Flores L, Gutierrez R, Madrid JF, et al. Pericytes. Morphofunction, interactions and pathology in a quiescent and activated mesenchymal cell niche. Histol Histopathol. 2009; 24: 909-69.

29. Diaz-Flores L, Gutierrez R, Garcia MP, et al. CD34+ stromal cells/fibroblasts/fibrocytes/telocytes as a tissue reserve and a principal source of mesenchymal cells. Location, morphology, function and role in pathology. Histol Histopathol. 2014; 29: 831-70.
30. Miranville A, Heeschen C, Sengenes C, et al. Improvement of postnatal neovascularization by human adipose tissue-derived stem cells. Circulation. 2004; 110: 349-55.

31. Suga H, Eto H, Inoue $\mathrm{K}$, et al. Cellular and molecular features of lipoma tissue: comparison with normal adipose tissue. Br J Dermatol. 2009; 161: 819-25. 